\title{
Spatial confinement of muonium atoms
}

\author{
K. S. Khaw, ${ }^{1, *}$ A. Antognini, ${ }^{1,2}$ T. Prokscha,${ }^{3}$ K. Kirch, ${ }^{1,2}$ L. Liszkay, ${ }^{4}$ Z. Salman, ${ }^{3}$ and P. Crivelli ${ }^{1, \dagger}$ \\ ${ }^{1}$ Institute for Particle Physics, ETH Zurich, 8093 Hoenggerberg, Switzerland \\ ${ }^{2}$ Laboratory for Particle Physics, Paul Scherrer Institute, 5232 Villigen PSI, Switzerland \\ ${ }^{3}$ Laboratory for Muon Spin Spectroscopy, Paul Scherrer Institute, 5232 Villigen PSI, Switzerland \\ ${ }^{4}$ IRFU, CEA, University Paris-Saclay F-91191 Gif-sur-Yvette Cedex, France
}

(Received 27 June 2016; published 30 August 2016)

\begin{abstract}
We report the achievement of spatial confinement of muonium atoms (the bound state of a positive muon and an electron). Muonium emitted into a vacuum from mesoporous silica reflects between two $\mathrm{SiO}_{2}$ confining surfaces separated by $1 \mathrm{~mm}$. From the data, one can extract that the reflection probability on the confining surfaces kept at $100 \mathrm{~K}$ is about $90 \%$ and the reflection process is well described by a cosine law. This technique enables new experiments with this exotic atomic system and is a very important step towards a measurement of the $1 S-2 S$ transition frequency using continuous-wave laser spectroscopy.
\end{abstract}

DOI: 10.1103/PhysRevA.94.022716

\section{INTRODUCTION}

Since its discovery by Hughes et al. [1], muonium (Mu), the bound state of an electron and a positive muon, has been a subject of extensive research. Being made of two leptons, muonium is an ideal system in which to study boundstate QED free of finite-size effects and in which hadronic corrections are strongly suppressed compared to hydrogen [2]. The study of its properties has led to the determination of the fine-structure constant (now best known by the electron $g-2$ measurement [3]), the mass and magnetic moment of the muon, and the best verification of charge equality in the first two generations of particles [4,5]. Muonium has also been used for searches of new physics [6] and applications for it in materials science have been found [7]. Due to the limited lifetime of the muon $(2.2 \mu \mathrm{s})$, muonium is unstable. It is produced by combining $\mu^{+}$from a beam with an electron of a target material. New experiments with this atomic system would benefit enormously from more intense and brighter sources and more efficient converters of the primary beam into muonium [8].

Recently, a sizable fraction of thermalized muonium emitted into a vacuum from mesoporous thin $\mathrm{SiO}_{2}$ films has been reported [9]. The muonium vacuum yield per implanted $\mu^{+}$ with $5 \mathrm{keV}$ implantation energy was measured to be $0.38(4)$ at $250 \mathrm{~K}$ and $0.20(4)$ at $100 \mathrm{~K}$. The high muonium vacuum yield, even at low temperatures, is an important step towards new measurements with this atomic system and especially towards a more precise measurement of the muonium $1 S-2 S$ transition frequency [5]. In fact, such a source of cold muonium (100 K compared to $300 \mathrm{~K}$ of the previous measurements) opens the possibility of performing continuous-wave (cw) laser spectroscopy of this transition because the larger vacuum yield compared to what has been previously reported [10-13] and the increase in the interaction time between the atoms and the laser beam will compensate for the lower power available compared to a pulsed laser. In fact, in the resonant weak-field

\footnotetext{
*Present address: Department of Physics, University of Washington, Seattle, WA 98195, USA.

†crivelli@phys.ethz.ch
}

approximation the excitation probability is proportional to the interaction time squared. Continuous-wave spectroscopy would decrease the statistical and systematical uncertainties of the previous experiment [5] since the broadening due to the laser chirp, the ac Stark effect, and the residual first-order Doppler shift related to pulsed laser spectroscopy would be eliminated. These effects resulted in a linewidth of about $20 \mathrm{MHz}$. Monte-Carlo simulations of the atoms' trajectories with numerical integration of the Bloch equations including photoionization and the ac Stark effect [14] show that the expected linewidth for a cw experiment is at the level of $1 \mathrm{MHz}$, approaching the $145-\mathrm{kHz}$ natural linewidth of the $1 S-2 S$ transition in muonium. The main source of broadening is due to the mean finite interaction time of the atoms with the laser beam which for the expected laser beam waist $\omega_{0}=200$ $\mu \mathrm{m}$ and a mean muonium velocity at $100 \mathrm{~K}$ of $v_{M}=2200 \mathrm{~m} / \mathrm{s}$ will be about $130 \mathrm{~ns}$. An enhancement cavity like that in use at the Max-Planck Institute of Quantum Optics in Garching for hydrogen excitation in the $2 S$ state [15] is used to generate $4 \mathrm{~W}$ of laser power at $244 \mathrm{~nm}$. The use of the cavity will grant a high degree of collinearity of the counterpropagating photons, thus reducing the first-order Doppler shift to a negligible level [15]. The broadening due to the ac Stark effect for the expected laser intensity $I$ can be estimated to be $\Delta v_{\mathrm{AC}}=1.67 \mathrm{I} \mathrm{Hz} \mathrm{cm}^{2} / \mathrm{W}=17 \mathrm{kHz}$.

In this paper, we demonstrate the feasibility of muonium confinement in a small volume or channel that allows one to perform such a measurement with currently available technology. Muonium confinement has the advantage of increasing the number of atoms crossing the laser beam if this is positioned along the axis of the channel (see Fig. 1). In this way the probability to laser excite a muonium atom is substantially enhanced, leading to an improvement in the signal rate. A similar scheme has been recently employed for positronium (Ps, the electron-positron bound state) to detect Ps atoms excited in the $2 S$ states $[16,17]$.

With the Low Energy Muon (LEM) beam line at PSI and the $\mu^{+}$-Mu converter geometry we report here, an improvement in precision of the $1 S-2 S$ transition frequency of more than an order of magnitude is in reach with a commercially available UV source [18] combined with an enhancement cavity. 


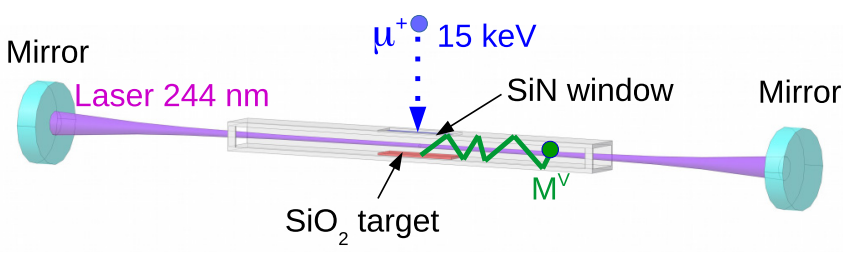

FIG. 1. Sketch of the muonium excitation in a confining volume. Muonium exiting the thin $\mathrm{SiO}_{2}$ film into vacuum $\left(\mathrm{M}^{\mathrm{V}}\right)$ is forced to bounce back and forth between the confining walls and thus multipassages through the laser beam can occur, enhancing its excitation probability.

\section{EXPERIMENTAL TECHNIQUE AND SETUP}

The setup used to test the $\mathrm{Mu}$ confinement is sketched in Fig. 2. It consisted of two aluminum thin plates of 0.5 and $1.0 \mathrm{~mm}$ sandwiching a $3 \times 3$ array of 50 -nm-thick $5.6 \times$ $5.6 \mathrm{~mm}^{2} \mathrm{SiN}$ membranes (Silson, Blisworth, UK) that had 0.2mm support ribs [see Fig. 2 (inset)]. To implant the keV muons in the confining cavity we used $\mathrm{SiN}$ windows that are quite robust (e.g., compared to carbon foils) and can be easily coated. The SiN membrane had an overall area of $17.6 \times 17.6 \mathrm{~mm}^{2}$ and an average transmission of $>80 \%$. The membrane was installed in front of the mesoporous $\mathrm{SiO}_{2}$ thin film which was glued to the sample holder. Pillars with adjustable heights were used to vary the distance $(h)$ between the mesoporous silica and the $\mathrm{SiN}$ window. The aluminum supports were coated with 3-4 nm of $\mathrm{SiO}_{2}$ on the side where $\mathrm{Mu}$ was confined. To avoid charging, the SiN window was coated with the same thickness of gold on the side of the incoming muons. The $\mathrm{Cu}$ sample holder was cooled down to $20 \mathrm{~K}$ repeatedly, and no mechanical damage due to thermal stress was observed on the $\mathrm{SiN}$ membrane.

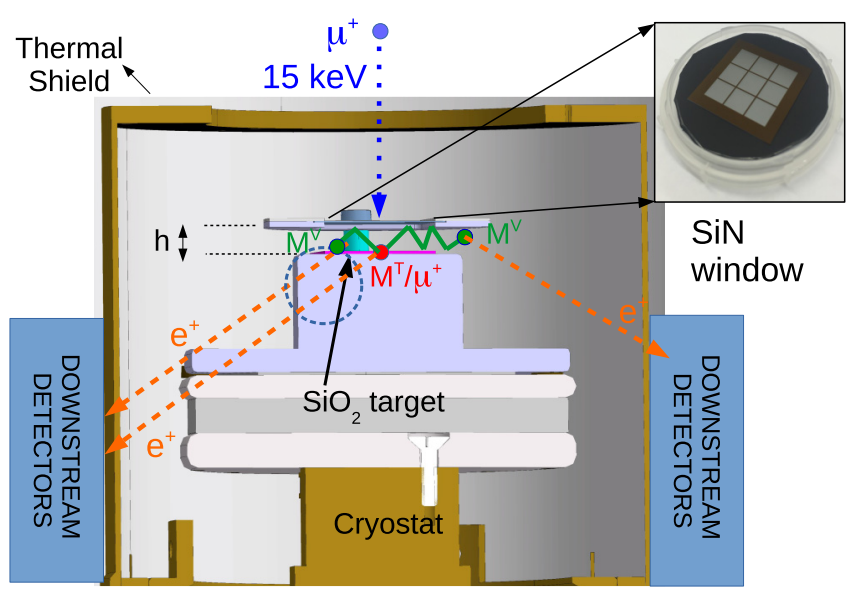

FIG. 2. Confinement of muonium between two surfaces. Sketch of the experimental setup and principle of the positron shielding technique technique. After crossing the thin SiN entrance window (shown in the inset) the $15-\mathrm{keV} \mu^{+}$stop in the mesoporous $\mathrm{SiO}_{2}$ thin film. Positrons from muons or muonium $\left(\mathrm{M}^{\mathrm{T}}\right)$ decaying in the target have a constant detection probability. Mu exiting the target into the vacuum $\left(\mathrm{M}^{\mathrm{V}}\right)$ have an increased and time-dependent detection probability because of the shielding/shadowing effect of the copper sample holder [shown as a dotted (blue) circle].
The mesoporous sample used in this study is the same as the one we measured previously [9] for which the Mu vacuum yield $F_{\mathrm{M}}^{\mathrm{vac}}$ was $40 \%$ at $250 \mathrm{~K}$ and $20 \%$ at $100 \mathrm{~K}$. As we have shown, the best fit to the data was achieved modeling $\mathrm{Mu}$ emission into a vacuum with a cosine angular distribution and a Maxwell-Boltzmann kinetic energy according to the sample temperature. At $T=20 \mathrm{~K}$, the Mu decays in the target because at this temperature it is adsorbed (sticks) to the $\mathrm{SiO}_{2}$ surface and therefore does not diffuse into the vacuum $[9,19,20]$. These parameters were used to simulate the $\mathrm{Mu}$ production in the mesoporous sample.

The principle of the measurements to test the muonium spatial confinement between two surfaces is sketched in Fig. 2. A fraction of the low-energy $\mu^{+}$from the LEM beam line [21] $\left(5000 \mu^{+} / \mathrm{s}\right.$ with a beam size of $\left.5 \mathrm{~mm}\right)$ are implanted through the thin $(50 \mathrm{~nm}) \mathrm{SiN}$ entrance window and stop in the mesoporous thin-film where about $60 \%$ of them form muonium in the bulk material $[9,22,23]$. TrimSP simulations, validated with previous measurements with muons [24], predict that about $96 \%$ of the $\mu^{+}$with an initial energy of $15 \mathrm{keV}$ are transmitted through the $\mathrm{SiN}$ window and implanted in the mesoporous silica $\left(\rho=1.1 \mathrm{~g} / \mathrm{cm}^{3}\right)$ with a mean energy of $4.9 \mathrm{keV}(1.0 \mathrm{keV}$ rms) which corresponds to a mean implantation depth of about $80 \mathrm{~nm}$ (26 nm rms). The formed muonium atoms diffuse through the interconnected porous network and a fraction of them reach the thin-film surface before the muon decays and are emitted into the vacuum $\left(10^{-9} \mathrm{mbar}\right)$ where muonium is free to move until it reaches the confining surface surrounding the target. The main goal of this work is to study the effect of the muonium hitting the walls in order to assess the feasibility of its confinement. If reflections at the walls occur, the $\mathrm{Mu}$ atoms will diffuse from the center of the setup in the radial direction. Therefore with increasing time, they approach the plastic scintillators which surround the sample plate used to detect the positrons from the muon decays (the downstream detectors shown in Fig. 2, see Ref. [21] for more details).

The positrons emitted from muonium which traveled a larger radial distance from the target before decaying have a considerably higher probability to be detected in the downstream positron counter because of the reduced shielding by the copper sample plate as schematically illustrated in Fig. 2. Therefore, if muonium diffuses the time spectra of the downstream detector are distorted as a consequence of the position-dependent detection probability as shown in Fig. 3. These are typical positron-shielding-technique time spectra simulated with GEANT4 [25] that were observed in Ref. [9]. The geometry of the copper sample holder was optimized in order to enhance the contrast for $\mathrm{Mu}$ atoms emitted into the vacuum compared to $\mathrm{Mu}$ or $\mu^{+}$decaying in the sample (see Fig. 2); i.e., compared to the standard sample holder used in the LEM beam line for muon-spin rotation the sample position was moved by $19 \mathrm{~mm}$ downstream and the holder diameter was reduced by a factor 2 [26,27].

In order to better visualize the effect of muonium atoms emitted into the vacuum and reflecting between the $\mathrm{SiN}$ window and the mesoporous $\mathrm{SiO}_{2}$, the simulated positron time spectra are divided by $e^{-t / \tau_{\mu}}$ to eliminate the muon lifetime $\left(\tau_{\mu}=2.2 \mu \mathrm{s}\right)$ effect as shown in Fig. 3 .

For $\mu^{+}$or muonium stationary on the sample plate, the detection efficiency of the decay positron as seen from the 


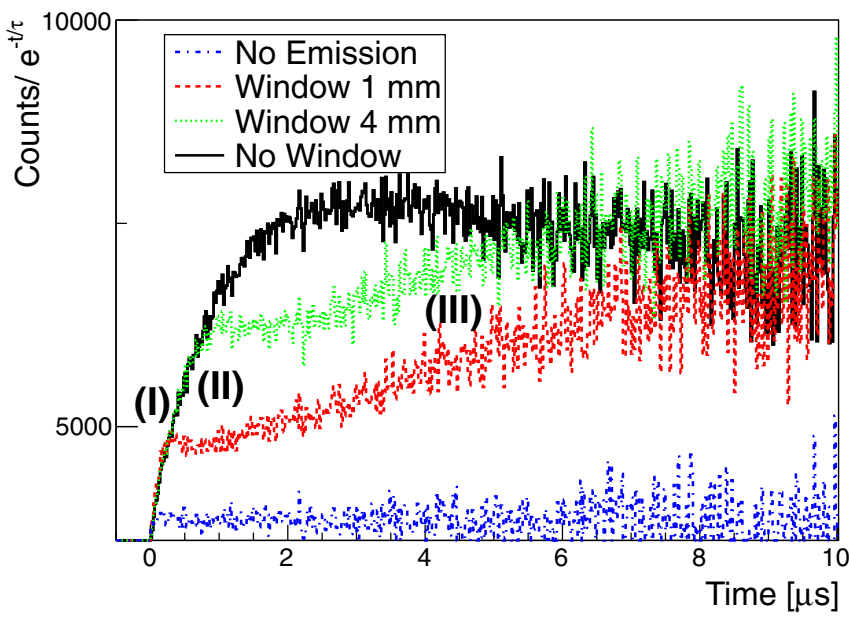

FIG. 3. Simulated time spectra of the downstream detectors after subtraction of the muon-decay for different cases: (i) Dotteddashed/blue line: No M emission into vacuum, (ii) Dotted/red line: emission of $\mathrm{Mu}$ into vacuum at $250 \mathrm{~K}$ and cosine reflection at the walls with $h=1 \mathrm{~mm}$ separation between the SiN entrance window and the mesoporous $\mathrm{SiO}_{2}$ thin film. (iii) Green/dashed line: same as (ii) but with $h=4 \mathrm{~mm}$. (iv) $\mathrm{M}$ Emission into vacuum at $250 \mathrm{~K}$ without $\mathrm{SiN}$ window. The three regions (I, II, III) defined on the graph are described in detail in the text.

downstream detector remains constant in time. Hence a straight horizontal line is expected as shown in Fig. 3 [dashed-dotted (blue) histogram]. For $\mathrm{Mu}$ emission into the vacuum without the $\mathrm{SiN}$ entrance window, an increase in the spectra is expected (solid/black histogram) because of the smaller shielding effect of the sample holder for muonium exiting the target.

When a SiN window is installed, the time evolution is more complicated. After emission into the vacuum, the $\mathrm{M}$ atoms propagate freely until they reach the $\mathrm{SiN}$ membrane. Therefore, the time spectra in the first few hundred nanoseconds [in region (I) in Fig. 3] are "identical" to the histograms computed without the window. When they reach the window, the increase in the detection efficiency stops because the muonium atoms are either adsorbed at the wall or back-reflected. If adsorption occurs, a flat curve is obtained (see the black solid curve in Fig. 4). If back-reflection occurs this results first in a sudden drop in positron counts as visible in region (II) of Figs. 3 and 4 because the muonium atoms move back to the sample holder plate where the shielding for the decaying positrons is higher. With time the muonium atoms will radially drift towards the surrounding positron detectors causing the increase in the time spectra at later times [region (III)].

From the increase related with this slow diffusion one can derive information on the reflection process and the adsorption probability. To illustrate the sensitivity to the reflection model different reflection scenarios of the muonium atoms at the confining walls, i.e., specular, isotropic, and reflections distributed according to a cosine distribution, were simulated (see Fig. 4).

\section{RESULTS}

The positron time spectra of the downstream detectors for measurements done at $h=1 \mathrm{~mm}$ and $h=4 \mathrm{~mm}$ separation

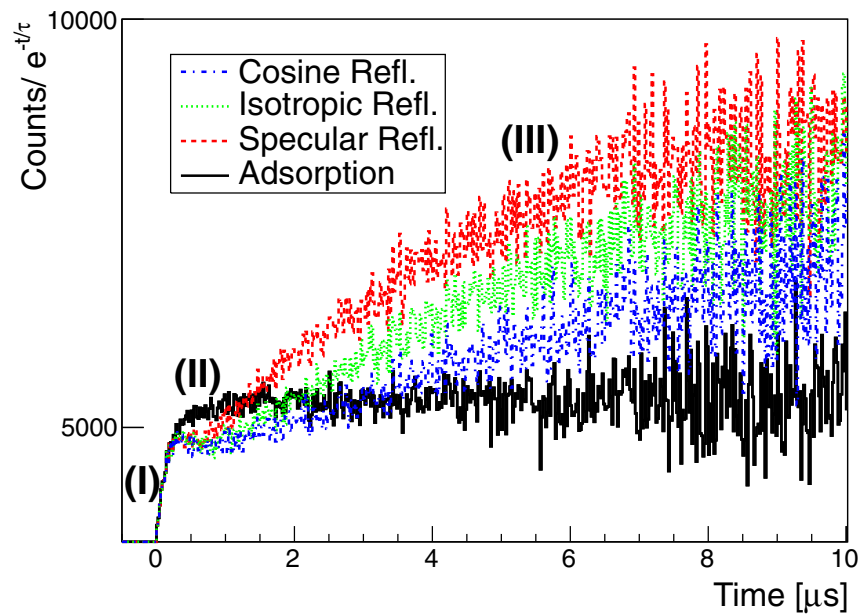

FIG. 4. Simulated time spectra for different reflection scenarios of muonium emitted into the vacuum at $250 \mathrm{~K}$ : no reflection, i.e. adsorption or sticking of muonium on the confining walls (black solid curve), specular ([dashed (red) curve], isotropic [dotted (green) curve], and cosine distributed relative to the surface normal [dotdashed (blue) curve] reflections.

and various temperatures are shown in Fig. 5. The solid line represents the simulated time spectra which result in a good agreement with the data. As expected from our previous results [9], the yield of muonium emitted into the vacuum increases with temperature; i.e., in the simulation the $F_{\mathrm{M}}^{\mathrm{vac}}$ is $20 \%$ at $100 \mathrm{~K}$ and $40 \%$ at $250 \mathrm{~K}$. For times $t \in[0 ; 0.5] \mu \mathrm{s}$ the time spectra increase quickly until a "plateau" is reached. This "plateau" level is proportional to the muonium emission probability into vacuum $F_{\mathrm{M}}^{\mathrm{vac}}$, whereas the linear coefficient of the slope at early times depends on the initial velocity of the muonium atoms and therefore on the sample temperature [9]. The time when the muonium atoms hit the $\mathrm{SiN}$ window is easily visible in the time spectra and increases, as one would expect, with the distance between the mesoporous silica and the entrance window. This is about $0.25 \mu \mathrm{s}$ for $h=1 \mathrm{~mm}$ and $1 \mu \mathrm{s}$ for $h=4 \mathrm{~mm}$. For later times, $t \in[1 ; 10] \mu \mathrm{s}$, the histograms show a slow increase. This demonstrates clearly that the muonium atoms can radially diffuse inside the confinement region and survive the collisions with the walls. This is nicely evidenced by the comparison with the $20 \mathrm{~K}$ data shown in Fig. 5 where Mu is produced but it does not diffuse out of the sample [9] because it sticks to the $\mathrm{SiO}_{2}$ surface. Hence, the data down to a temperature of $100 \mathrm{~K}$ clearly indicate $\mathrm{Mu}$ bouncing between the two walls and thus the feasibility of its confinement in a small volume.

The increase at later times depends not only on the muonium kinetic energy distribution but also on the type of reflection and on the reflection probability. Thus the slope at delayed times contains information on the reflection process and its probability $R$. The best fits to the data are obtained by simulating a reflection following a cosine distribution (see Fig. 6) as predicted by the kinetic theory of gases [28] and supported by recent advanced simulations of atoms' reflections at rough surfaces [29]. Assuming this is the correct distribution, probabilities of $R_{250 \mathrm{~K}}=94 \pm 4 \%$ at $250 \mathrm{~K}$ and $R_{100 \mathrm{~K}}=$ $90 \pm 6 \%$ at $100 \mathrm{~K}$ are extracted. These reflection probabilities 

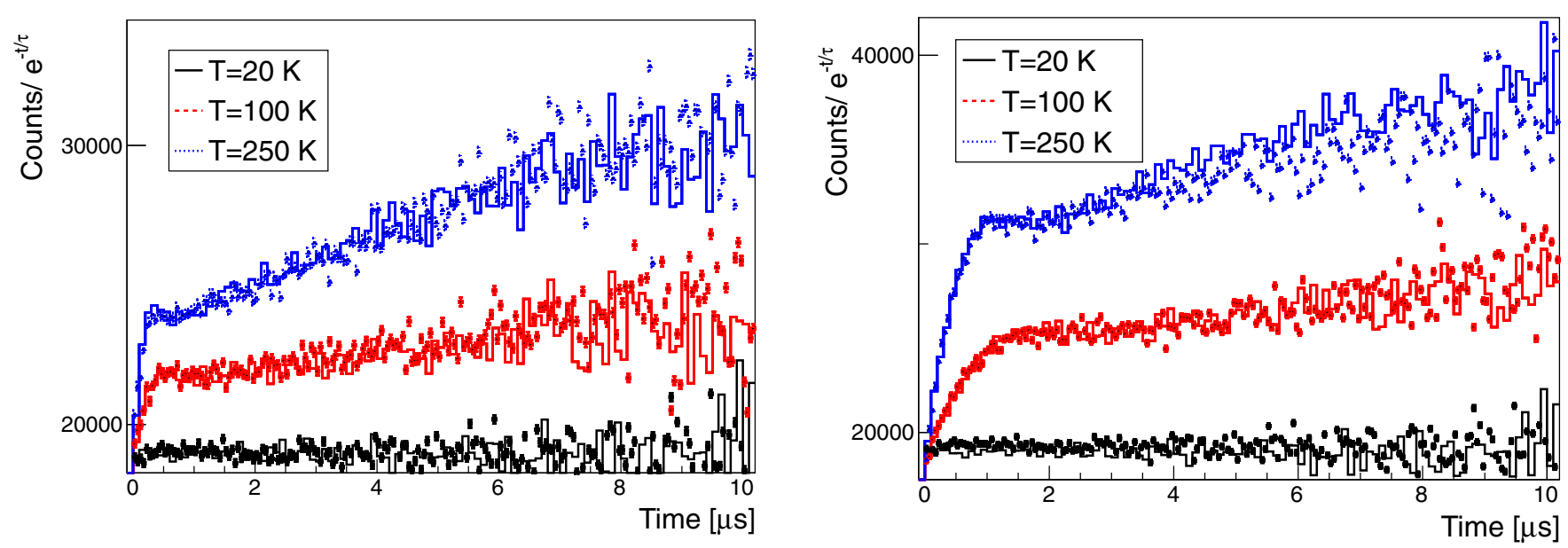

FIG. 5. The points are the measured time spectra at $T=20,100$, and $250 \mathrm{~K}$ for a distance between the $\mathrm{SiO}_{2}$ target and the $\mathrm{SiN}$ window of $h=1 \mathrm{~mm}$ (left) and of $h=4 \mathrm{~mm}$ (right). The solid lines are the corresponding simulated time spectra (see text for more details).

are compatible with the fraction of atoms with kinetic energies below about 4-5 meV for muonium emitted with Maxwell Boltzmann distributions at $100 \mathrm{~K}$ and $250 \mathrm{~K}$, respectively. This energy corresponds to about $50 \mathrm{~K}$, a substrate temperature at which muonium is known to stick to $\mathrm{SiO}_{2}$. This effect was first observed in silica powder by measuring the hyperfine splitting (HFS) as a function of the temperature. It was noticed that below $100 \mathrm{~K}$ the value of the HFS was dropping from the vacuum value and it was shown that this was consistent with thermal adsorption at the $\mathrm{SiO}_{2}$ [20]. In mesoporous silica films, an abrupt drop of the Mu emitted into the vacuum below $100 \mathrm{~K}$ has been observed. Because the muon-spin rotation data show a constant muonium formation probability as a function of the temperature, this was also interpreted as adsorption of $\mathrm{Mu}$ atoms on the surface of the pores [9]. In light of those results, the efficient reflection of $\mathrm{Mu}$ with energies of 4-5 meV on $\mathrm{SiO}_{2}$ surfaces could be anticipated. A possible explanation for the observed reflection probabilities (summarized in Table I) is that muonium atoms with kinetic energies smaller than the

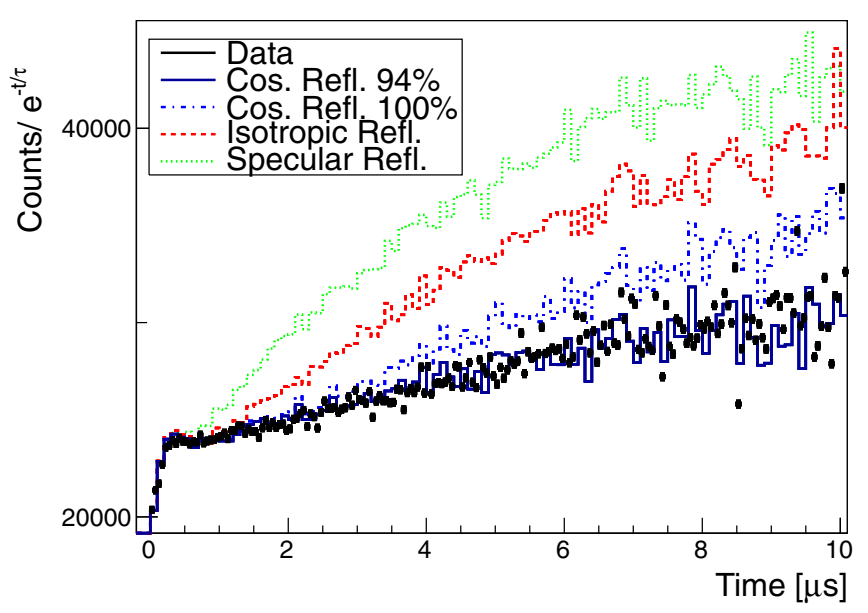

FIG. 6. The points are the data at $250 \mathrm{~K}$ for a distance between the $\mathrm{SiO}_{2}$ target and the $\mathrm{SiN}$ window of $h=1 \mathrm{~mm}$. The lines are the simulated time spectra for different reflection scenarios (see text for more details). depth of the Lennard-Jones potential (which can be estimated with our previous results) are adsorbed at the $\mathrm{SiO}_{2}$ walls also at the respective higher substrate temperatures.

\section{SUMMARY AND CONCLUSIONS}

The comparison of the data and the simulation shows that the reflection effect of muonium between the confining surfaces is very significant compared to the adsorption effect and follows a cosine distribution. The measured time spectra show that muonium atoms are reflected from confining surfaces even at $100 \mathrm{~K}$. At this temperature and for a distance of $h=1 \mathrm{~mm}$, this is estimated to give an enhancement of a factor 5 in the excitation probability for the muonium $1 S-2 S$ transition compared to a geometry in which the atoms would just pass only once through the laser beam. Therefore, the possibility of muonium confinement, which has been demonstrated here, opens the way to a measurement of the muonium $1 S$ - $2 S$ transition frequency with existing technology at the $0.2 \mathrm{ppb}$ level (an improvement of a factor 20 compared to Ref. [5]). This will provide a test of bound-state QED (the theoretical uncertainty is $0.4 \mathrm{ppb}[30,31]$ ), the best verification of charge equality in the first two generations of particles, and an improved determination of the muon mass at the $40 \mathrm{ppb}$ level (a factor 3 better than currently determined [4]). Such a measurement would be limited by the statistical uncertainty and therefore an even higher accuracy could be expected in the near future because of the ongoing efforts to develop high-flux and -brightness slow muon beams [32,33].

TABLE I. Summary of the number of the reflection probabilities at the surface and the production of confined $\mathrm{Mu}$ atoms (1/s) using the LEM beam line and the target geometry of Fig. 2 for different temperatures.

\begin{tabular}{lcc}
\hline \hline Temperature $(\mathrm{K})$ & $\mathrm{Mu}(1 / \mathrm{s})$ & Refl. prob. $(\%)$ \\
\hline 20 & 0 & 0 \\
100 & $768 \pm 31$ & $90 \pm 6$ \\
250 & $1459 \pm 58$ & $94 \pm 4$ \\
\hline \hline
\end{tabular}




\section{ACKNOWLEDGMENTS}

This work has been supported by the Swiss National Science Foundation under Grants No. 200020_166286 and No. 200020_159754 and by ETH Zurich Research Grant
No. ETH-35 14-1. We also acknowledge the help of the PSI and ETH Zurich IPP workshops and support groups. Special thanks to A. Gendotti, M. Horisberger, and A. Suter. The LEM measurements were performed at the Swiss Muon Source $\mathrm{S} \mu \mathrm{S}$, PSI.
[1] V. W. Hughes, D. W. McColm, K. Ziock, and R. Prepost, Phys. Rev. Lett. 5, 63 (1960).

[2] S. G. Karshenboim, Phys. Rep. 422, 1 (2005).

[3] G. Gabrielse, D. Hanneke, T. Kinoshita, M. Nio, and B. Odom, Phys. Rev. Lett. 97, 030802 (2006).

[4] W. Liu, M. G. Boshier, S. Dhawan, O. van Dyck, P. Egan, X. Fei, M. Grosse Perdekamp, V. W. Hughes, M. Janousch, K. Jungmann, D. Kawall, F. G. Mariam, C. Pillai, R. Prigl, G. zu Putlitz, I. Reinhard, W. Schwarz, P. A. Thompson, and K. A. Woodle, Phys. Rev. Lett. 82, 711 (1999).

[5] V. Meyer, S. N. Bagayev, P. E. G. Baird, P. Bakule, M. G. Boshier, A. Breitruck, S. L. Cornish, S. Dychkov, G. H. Eaton, A. Grossmann, D. Hubl, V. W. Hughes, K. Jungmann, I. C. Lane, Y. W. Liu, D. Lucas, Y. Matyugin, J. Merkel, G. zuPutlitz, I. Reinhard, P. G. H. Sandars, R. Santra, P. V. Schmidt, C. A. Scott, W. T. Toner, M. Towrie, K. Trager, L. Willmann, and V. Yakhontov, Phys. Rev. Lett. 84, 1136 (2000).

[6] L. Willmann, P. V. Schmidt, H. P. Wirtz, R. Abela, V. Baranov, J. Bagaturia, W. Bertl, R. Engfer, A. Grossmann, V. W. Hughes, K. Jungmann, V. Karpuchin, I. Kisel, A. Korenchenko, S. Korenchenko, N. Kravchuk, N. Kuchinsky, A. Leuschner, V. Meyer, J. Merkel, A. Moiseenko, D. Mzavia, G. zuPutlitz, W. Reichart, I. Reinhard, D. Renker, T. Sakhelashvilli, K. Trager, and H. K. Walter, Phys. Rev. Lett. 82, 49 (1999).

[7] A. Yaouanc and P. Dalmas de Reotier, Muon Spin Rotation, Relaxation, and Resonance: Applications to Condensed Matter (Oxford University, Oxford, UK, 2011).

[8] K. Jungmann, Nucl. Phys. B 155, 355 (2006); J. Phys. Soc. Jpn. 155, 355 (2016).

[9] A. Antognini, P. Crivelli, T. Prokscha, K. S. Khaw, B. Barbiellini, L. Liszkay, K. Kirch, K. Kwuida, E. Morenzoni, F. M. Piegsa, Z. Salman, and A. Suter, Phys. Rev. Lett. 108, 143401 (2012).

[10] A. P. Mills, J. Imazato, S. Saitoh, A. Uedono, Y. Kawashima, and K. Nagamine, Phys. Rev. Lett. 56, 1463 (1986).

[11] G. M. Marshall, J. B. Warren, D. M. Garner, G. S. Clark, J. H. Brewer, and D. G. Fleming, Phys. Lett. A 65, 351 (1978).

[12] G. A. Beer, G. M. Marshall, G. R. Mason, A. Olin, Z. Gelbart, K. R. Kendall, T. Bowen, P. G. Halverson, A. E. Pifer, C. A. Fry, J. B. Warren, and A. R. Kunselman, Phys. Rev. Lett. 57, 671 (1986).

[13] A. C. Janissen, G. A. Beer, G. R. Mason, A. Olin, T. M. Huber, A. R. Kunselman, T. Bowen, P. G. Halverson, C. A. Fry, K. R. Kendall, G. M. Marshall, and J. B. Warren, Phys. Rev. A 42, 161 (1990).

[14] M. Haas, U. D. Jentschura, C. H. Keitel, N. Kolachevsky, M. Herrmann, P. Fendel, M. Fischer, T. Udem, R. Holzwarth,
T. W. Hänsch, M. O. Scully, and G. S. Agarwal, Phys. Rev. A 73, 052501 (2006).

[15] C. G. Parthey, A. Matveev, J. Alnis, B. Bernhardt, A. Beyer, R. Holzwarth, A. Maistrou, R. Pohl, K. Predehl, T. Udem, T. Wilken, N. Kolachevsky, M. Abgrall, D. Rovera, C. Salomon, P. Laurent, and T. W. Hänsch, Phys. Rev. Lett. 107, 203001 (2011); A. Beyer, J. Alnis, K. Khabarova, A. Matveev, C. G. Parthey, D. C. Yost, R. Pohl, T. Udem, T. W. Haensch, and N. Kolachevsky, Ann. Phys. 525, 671 (2013).

[16] D. Cooke, P. Crivelli, J. Alnis, A. Antognini, B. Brown, S. Friedreich, A. Gabard, T. W. Haensch, K. Kirch, A. Rubbia, and V. Vrankovic, Hyperfine Int. 233, 67 (2015).

[17] P. Crivelli, D. Cooke, and S. Friedreich, Int. J. Mod. Phys.: Conf. Ser. 30, 1460257 (2014).

[18] TOPTICA photonics, http://www.toptica.com/.

[19] D. R. Harshman, R. Keitel, M. Senba, R. F. Kiefl, E. J. Ansaldo, and J. H. Brewer, Phys. Lett. A 104, 472 (1984).

[20] R. F. Kiefl, B. D. Patterson, E. Holzschuh, W. Odermatt, and D. R. Harshman, Hyperfine Int. 17, 563 (1984).

[21] T. Prokscha, E. Morenzoni, K. Deiters, F. Foroughi, D. George, R. Kobler, A. Suter, and V. Vrankovic, Nucl. Instrum. Methods Phys. Res., Sect. A 595, 317 (2008).

[22] M. H. Dehn et al., J. Phys.: Conf. Ser. 551, 012006 (2014)

[23] P. Bakule et al., Prog. Theor. Exp. Phys. (2013) 103C01.

[24] E. Morenzoni, H. Glückler, T. Prokscha, R. Khasanov, H. Luetkens, M. Birke, E. M. Forgan, Ch. Niedermayer, and M. Pleines, Nucl. Instrum. Methods Phys. Res., Sect. B 192, 254 (2002).

[25] S. Agostinelli et al., Nucl. Instrum. Methods Phys. Res., Sect. A 506, 250 (2003).

[26] K. S. Khaw, A. Antognini, P. Crivelli, K. Kirch, E. Morenzoni, Z. Salman, A. Suter, and T. Prokscha, J. Instrum. 10, P10025 (2015).

[27] K. S. Khaw, PhD thesis, ETH Zurich, 2015, available online at http://dx.doi.org/10.3929/ethz-a-010486463.

[28] M. Knudsen, The Kinetic Theory of Gases (Methuen, London, 1934).

[29] F. Celestini and F. Mortessagne, Phys. Rev. E 77, 021202 (2008)

[30] K. Pachucki et al., J. Phys. B 29, 177 (1996).

[31] S. Karshenboim, Z. Phys. D 39, 109 (1997); Can. J. Phys. 77, 241 (1999).

[32] Y. Bao, A. Antognini, W. Bertl, M. Hildebrandt, K. S. Khaw, K. Kirch, A. Papa, C. Petitjean, F. M. Piegsa, S. Ritt, K. Sedlak, A. Stoykov, and D. Taqqu, Phys. Rev. Lett. 112, 224801 (2014).

[33] P. Strasser et al., J. Phys.: Conf. Ser. 551, 012065 (2014). 